\title{
Lung Ultrasonography for COVID-19 Patients in Out of Hospital Settings
}

\author{
Mahathar Abd Wahab ${ }^{1}$ [ . Elisa A. Eddie ${ }^{1}$. Ummar Qayyum Ahmad Ibrahim Ahmad ${ }^{2} \cdot$ Hidayah Shafie $^{1}$. \\ Sarah Binti Shaikh Abd Karim² . Shaik Farid Abdull Wahab ${ }^{3}$
}

Received: 6 April 2021 / Accepted: 6 June 2021 / Published online: 15 January 2022

○ Società Italiana di Ultrasonologia in Medicina e Biologia (SIUMB) 2022

\begin{abstract}
Purpose The portability of a hand-held ultrasound allows the health care worker to conduct lung ultrasound in out-of-hospital setting. It is used as a tool to conduct staging and triaging for COVID-19 patients. This study evaluated the utilization of lung ultrasound in an out-of-hospital setting versus chest x-rays in detecting and staging of COVID-19 patients with pneumonia. Methods The study was conducted among COVID-19 subjects at an out-of-hospital setting whereby lung ultrasound was done and subsequently chest $\mathrm{x}$-rays were taken after being admitted to the health care facilities. Lung ultrasound findings were reviewed by emergency physicians, while the chest x-rays were reviewed by radiologists. Radiologists were blinded by the patients' lung ultrasound findings and clinical conditions. The analysis of the agreement between the lung ultrasound findings and chest $\mathrm{x}$-rays was conducted.

Results A total of 261 subjects were recruited. LUS detected pulmonary infiltrative changes in more stage 3 COVID-19 subjects in comparison to chest X-rays. Multiple B-lines were the predominant findings at the right lower anterior, posterior and lateral zones. Interstitial consolidations and ground glass opacities were the predominant descriptive findings in chest $\mathrm{x}$-rays. However, there was no agreement between lung ultrasound and chest $\mathrm{x}$-ray findings in detecting COVID-19 pneumonia as the Cohen's Kappa coefficient was 0.08 (95\% CI 0.06-0.22, $p=0.16$ ).

Conclusion The diagnostic imaging and staging of COVID-19 patients using lung ultrasound in out-of-hospital settings showed LUS detected lung pleural disease more often than CXR for stage 3 COVID-19 patients.
\end{abstract}

Keywords COVID-19 · SARS-CoV-2 · Out-of-hospital · Chest X-rays · Lung ultrasound · COVID-19 staging

$\begin{array}{ll}\text { Abbreviations } \\ \text { ARDS } & \text { Acute Respiratory Distress Syndrome } \\ \text { CT } & \text { Computer Tomography } \\ \text { CXR } & \text { Chest x-ray } \\ \text { GGO } & \text { Ground glass opacity } \\ \text { ICU } & \text { Intensive Care Unit } \\ \text { LUS } & \text { Lung ultrasound } \\ \text { MOH } & \text { Ministry of Health }\end{array}$

Mahathar Abd Wahab

mahatharwahab@yahoo.com

Elisa A. Eddie

elisaeddie@gmail.com

Ummar Qayyum Ahmad Ibrahim Ahmad

ummarqayyum@gmail.com

Hidayah Shafie

heedaymd@yahoo.com

Sarah Binti Shaikh Abd Karim

sarahabdkarim@gmail.com
MREC Medical Research \& Ethics Committee

NMRR National Medical Research Register

POCUS Point-of-care ultrasound

PUS Patient under surveillance

RALE Radiographic assessment of lung edema

RT-PCR Reverse transcription polymerase chain reaction
Shaik Farid Abdull Wahab

drsfarid@usm.my

1 Department of Emergency and Trauma, Hospital Kuala Lumpur, Federal Territories Kuala Lumpur, Kuala Lumpur, Malaysia

2 Department of Emergency and Trauma, Hospital Sg Buloh, Sungai Buloh, Selangor Darul Ehsan, Malaysia

3 Department of Emergency Medicine, School of Medical Sciences, Universiti Sains Malaysia, Kubang Kerian, Kelantan, Malaysia 
SARS-CoV-2 Severe acute respiratory syndrome coronavirus 2

SPSS Statistical Package for the Social Sciences WHO World Health Organization

\section{Introduction}

A novel coronavirus disease outbreak (nCoV-19) began in December 2019 in Wuhan, China [1]. COVID-19 infection which is caused by severe acute respiratory syndrome coronavirus 2 (SARS-CoV-2), was declared on the 11th March 2020 by the World Health Organization (WHO) as a global pandemic. The SARS-CoV-2 virus mainly affects the lower respiratory system. The initial series of cases from Wuhan, China reported lower respiratory tract symptoms including fever, dry cough and dyspnea. Headache, dizziness, general weakness, vomiting and diarrhea have also been observed [2]. Approximately $80 \%$ of patients would have mild symptoms, about $14 \%$ experienced moderate to severe illness with $5 \%$ being critically ill [3].

Rapid spread of the disease with its high infectivity rate had imposed unprecedented challenges to the healthcare system, hence healthcare providers need to be adaptive with the latest guidelines and approaches for the management of COVID-19. The utilization of conventional tools such as the stethoscope carries a high risk of nosocomial transmission [4]. Moreover, the stethoscope and auscultation also have limited usage in assessing COVID-19 pneumonia with only a sensitivity of $19-67 \%$ and specificity of $36-96 \%$ on auscultation [5]. The use of portable chest X-ray (CXR) raises the issue of contamination, unless a dedicated machine is reserved for the patients. CXR moreover correlates poorly with the clinical picture as compared to computed tomography (CT) and ultrasound imaging [6]. The risk of critically unwell patients being transported for a CT scan followed by the requisite decontamination procedures makes this type of imaging dangerous and time consuming [7]. Lung ultrasound on the other hand minimizes medical devices contaminations as the hand-held ultrasound machine can be wrapped and easily disinfected [4].

COVID-19 pneumonia has been studied extensively and staged based on the duration of symptoms. Computerized tomography scan of the thorax is the gold standard in radiological imaging of COVID-19 patients. The typical characteristics found are thickened pleura, ground glass shadows, pulmonary infiltrating shadows, subpleural and trans-lobar consolidations affecting more than two lobes. Pleural effusion is rarely seen in COVID-19 thoracic CT scan findings. Several studies concluded that abnormal findings on the CT scan were correctly diagnosed with LUS with a sensitivity of $100 \%$, specificity of $78.6 \%$ and the LUS score correlated well with the CT Total Severity Score (CT TSS) $[8$,
9]. The characteristics of the LUS findings were described as thickened pleural line, B lines with multifocal, discrete or confluent patterns, small centromeric consolidations with multilobar distribution of abnormalities. Pleural effusion was also rare in LUS findings [10-12]. However, patients with preexisting interstitial or pleural lung disease should be interpreted with caution because the existing of B-lines and pleural irregularity are also LUS findings of interstitial lung disease. Comparison with patients baseline LUS findings, where available may aid physician in interpreting the findings. Thus, a positive LUS findings of pneumonia however does not diagnose COVID-19 but aids in the diagnosis of pneumonitis [9, 13]. A positive RT-PCR remains the reference standard in diagnosing COVID-19.

In Malaysia, quarantines centers have been established to contain a 'Person Under Surveillance' (PUS) hence preventing the spread of COVID-19 infections to the community. Besides quarantine centers, a large number of infected patients were also detected in immigration detention depots and prisons. These patients required admission to either a stepdown medical facility or a COVID-19 dedicated hospital with full intensive care monitoring capabilities. Mass screening and triaging were performed on positive COVID19 patients based on physiological parameters and lung ultrasound assessment to determine disease severity. Based on the severity, the patients were then either sent to a stepdown medical facility or a COVID-19 dedicated hospital with full intensive care monitoring capabilities.

Lung ultrasound usage is also used at an out-of-hospital setting such as screening centers. In combination with history and reverse transcription polymerase chain reaction (RT-PCR) assay, the role of ultrasound aids clinicians in screening and triaging, and increases diagnostic sensitivity of detecting pneumonia among COVID-19 patients [12]. Our primary objective is to compare the diagnostic imaging of COVID-19 staging using lung ultrasound in the out-ofhospital settings versus chest $\mathrm{x}$-ray (CXR) imaging. This study also describes the lung ultrasound patterns in clinical stage 3 COVID-19 pneumonia (symptomatic patient with clinical features of pneumonia) in comparison with the CXR.

\section{Materials and Methods}

This was an observational study conducted in out-of-hospital settings in a 3-month period (April 2020-June 2020) and was approved by the Medical Research and Ethics Committee (MREC) and registered with the National Medical Research Register (NMRR-20-1599-55711). 


\section{Subjects}

The study subjects were adult patients above 18 years old with COVID-19, diagnosed by reversed transcription polymerase chain reaction (RT-PCR) detected at quarantine centers, immigration detention depots and prisons. Exclusion criteria were pregnant and lactating women, cognitive impaired and critically ill patients.

\section{Image Acquisition}

Lung Ultrasound LUS was performed by trained pointof-care-ultrasound (POCUS) providers. All providers underwent a focused training on LUS to standardize the protocol and to optimize the quality of image acquisition. LUS was performed using 2 types of portable ultrasound machines: (a) Philips Lumify ${ }^{\circledR}$ Handheld Ultrasound, b) Ben $Q^{\circledR}$ T3300 Portable Ultrasound. Probe covers were used as part of infection control measure. A low frequency curvilinear probe was used to scan the patient's thorax in a sitting position, using Rouby's Protocol (Fig. 1) [14]. LUS was performed on six segments (anterior superior, anterior inferior, lateral superior, lateral inferior, posterior superior and posterior inferior) bilaterally. The sweep was performed from medial to lateral and downward. The probe was placed both longitudinally and transversely, with the probe marker pointing cephalad and medial, respectively. For each examination, B-mode evaluation was performed with serial images and clips were labeled, saved and archived. The images were later reviewed by another provider for verification. A scoring system based on the degree of loss of aeration was performed. The scores were; 0 points: A lines (normal aerated lung); 1 point (Figs. 2,
3): multiple B lines (arising from one point of the pleural line and from small peripheral consolidation and spreading down like rays maintaining their brightness until the edge of the screen without fading), either separated regularly or irregularly (moderate loss of aeration); 2 points: multiple coalescent B lines (severe loss of aeration); 3 points: lung consolidation, subpleural consolidation with irregular pleural line (complete loss of aeration). The sum of scores from all 12 lung windows represents the loss of aeration score and it is called the LUS score $[15,16]$. The LUS score is sub-categorized based on severity, $0=$ None; $1-7=$ Mild; $8-18=$ Moderate and $19-36=$ Severe [15].

Chest $X$-Ray CXRs were performed on all patients upon admission to health care facilities either at quarantine centers or at the hospital. All CXRs were reviewed and subsequently reported by radiologists who were blinded by patients' LUS findings and clinical conditions. The CXRs were then scored based on the Radiographic Assessment of Lung Edema (RALE) score [17]. RALE score was calculated as the summed products of the consolidation and density scores of each radiograph quadrant (right upper, right lower, left upper and left lower quadrants) with a maximum score of 12 for one quadrant and a maximum total score of 48 . A high baseline RALE score of $\geq 30$ is associated with decreased survival and worse outcomes; this is evident not only by the degree of hypoxemia, but also due to severely depressed compliance of respiratory system and the extent of lung epithelial injury [18]. RALE score is previously used to quantify the extent of lung involvement in ARDS and is currently applied in COVID19 pneumonia to standardize and objectively quantify the radiographic report and to produce a prognostic score at the time of the patient's admission.
Fig. 1 Lung ultrasound and Chest X-rays for Detection of Pulmonary Infiltrates. The number of confirmed Category 3 COVID-19 subjects $(n)$ with or without pulmonary infiltrates detected by chest X-ray or lung ultrasound is demonstrated

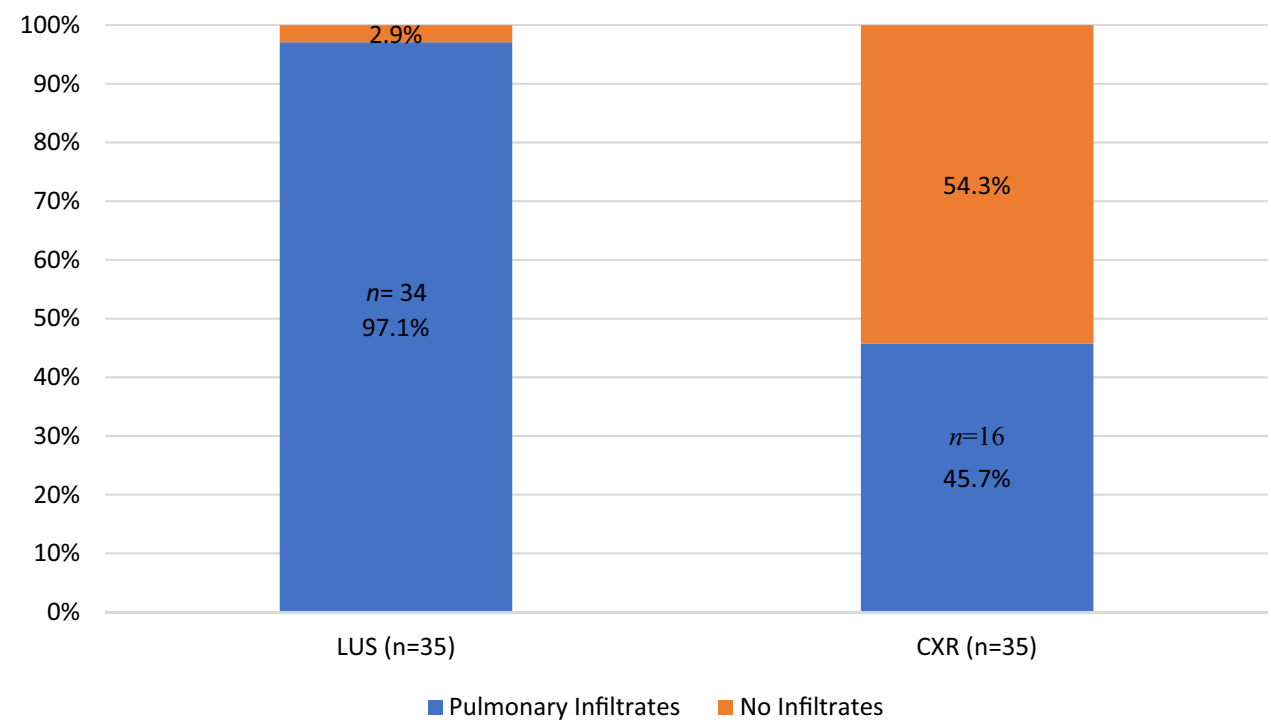


Fig. 2 Rouby's Protocol (12 lung-window) for LUS in suspected or confirmed COVID-19. A Right anterior zones, $\mathbf{B}$ right lateral zones, $\mathbf{C}$ Right posterior zones

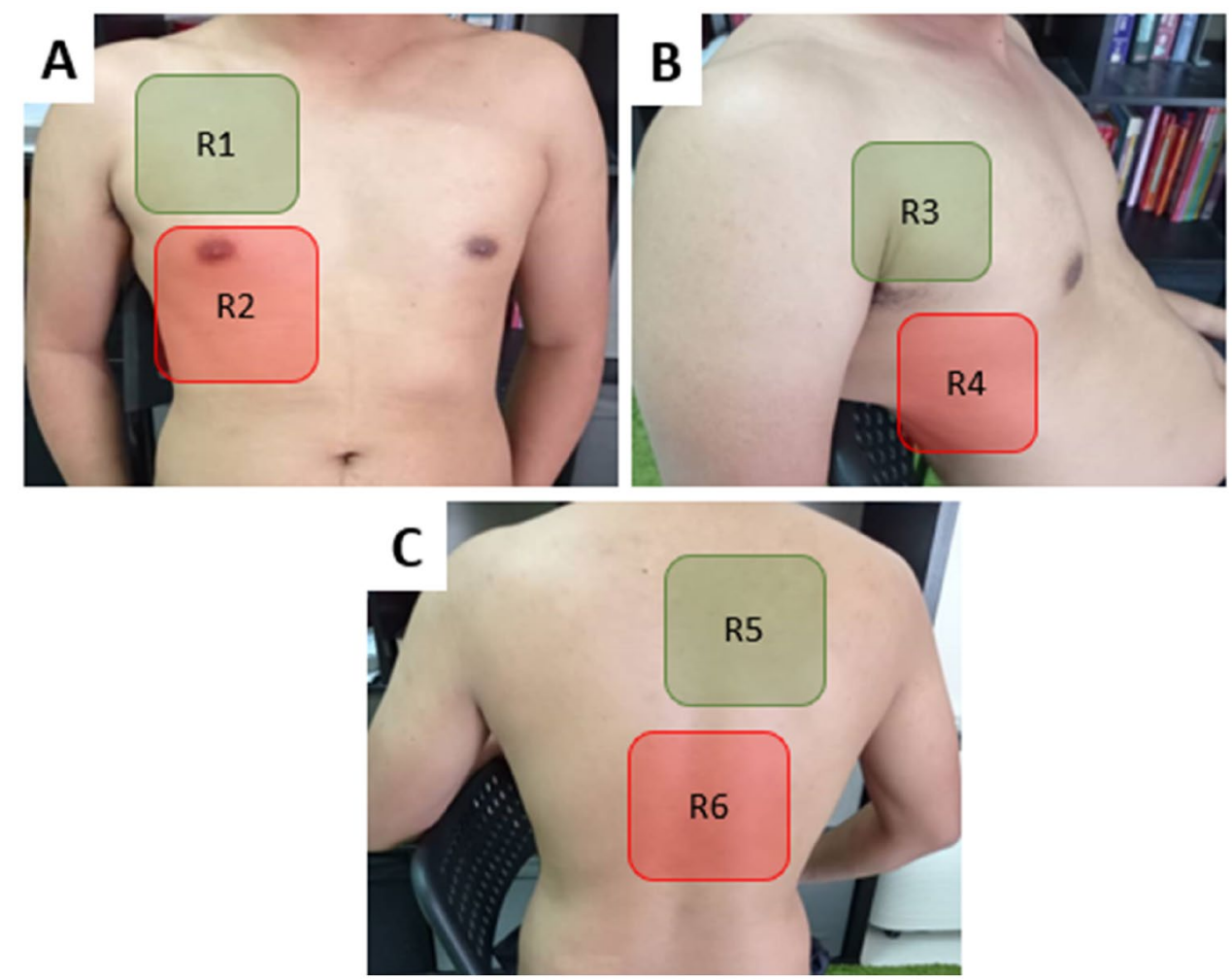

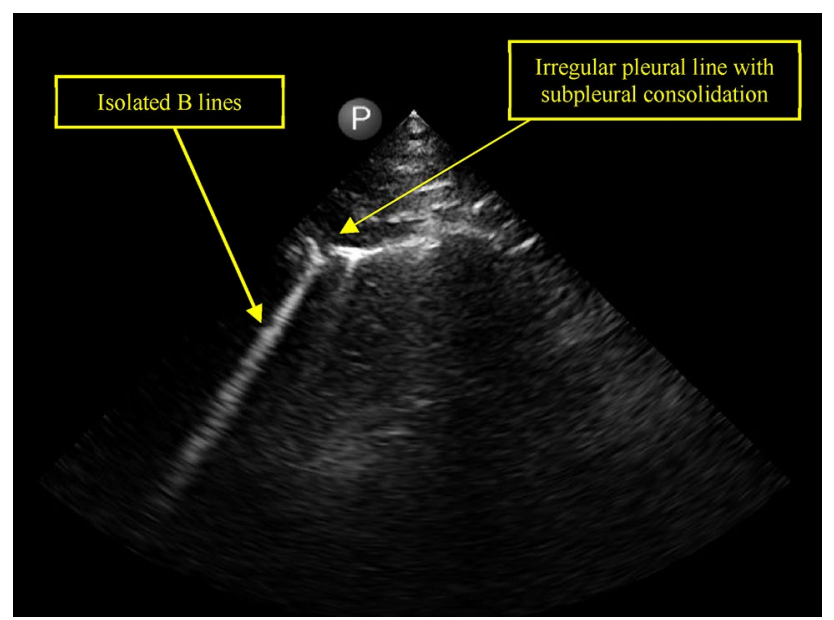

Fig. 3 Small peripheral localized subpleural consolidation, irregular pleural line appearance and isolated B lines; this represents moderate loss of aeration (Point: 1)

\section{Statistical Analysis}

The data analysis was done using SPSS version 22. CXR and LUS findings were classified into ordinal categories according to the scoring system for each diagnostic methods-normal, mild, moderate and severe. Quantitative data were summarized with mean \pm standard deviation (SD) or median (interquartile range). Qualitative data were summarized with number (\%). Chi square and Fisher's exact test were utilized and the agreement between LUS and CXR to interpret the presence of COVID-19 pneumonia was evaluated with Cohen's Kappa coefficient. Differences were considered statistically significant at the 2 -sided $P<0.05$ level.

\section{Results}

A total of two hundred and sixty-one subjects were recruited between $1^{\text {st }}$ April and $30^{\text {th }}$ June 2020.Characteristics of the subjects are presented in Table 1. The mean age of all subjects was 30 years and $96 \%$ were men. The most common comorbidities were diabetes mellitus and hypertension. $73.6 \%$ of the subjects were asymptomatic. Those who were symptomatic mainly had fever, cough and sore throat.

LUS assessment Two hundred and twenty-seven subjects had normal LUS findings. Among patients with stage 3 COVID-19, LUS detected pulmonary changes in more subjects than CXR (97\% vs 45\%). Vice versa, among subjects with normal LUS but reported abnormal CXR only 1 subject had pulmonary infiltrate changes (Fig. 1). Multiple B-lines were the predominant findings at the right lower anterior, posterior and lateral zones (Table 2).

Chest $x$-ray features Approximately $93.9 \%$ of chest radiographs were reported as normal and only 16 subjects (6.1\%) had abnormal findings. Interstitial consolidations were the predominant changes in $5 \%$ of the studied population while 
Table 1 Demographics and clinical characteristics of patients $(N=261)$

\begin{tabular}{|c|c|}
\hline \multicolumn{2}{|l|}{ Demographics } \\
\hline Gender (Male)- $N(\%)$ & $252(96.6)$ \\
\hline Gender (Female)- $N(\%)$ & $9(3.4)$ \\
\hline Age (years) mean (SD) & $30(11.64)$ \\
\hline \multicolumn{2}{|l|}{ Past Medical History } \\
\hline Cardiovascular disease- $N(\%)$ & $2(0.8)$ \\
\hline Pulmonary disease- $N(\%)$ & $4(1.5)$ \\
\hline Diabetes Mellitus- $N(\%)$ & $11(4.2)$ \\
\hline Chronic Kidney disease- $N(\%)$ & $1(0.4)$ \\
\hline Hypertension- $N(\%)$ & $16(6.1)$ \\
\hline Smoker- $N(\%)$ & $26(10.0)$ \\
\hline \multicolumn{2}{|l|}{ Symptoms } \\
\hline Dyspnea- $N(\%)$ & $3(1.1)$ \\
\hline Fever- $N(\%)$ & $45(17.2)$ \\
\hline Sore throat- $N(\%)$ & $21(8.0)$ \\
\hline Cough- $N(\%)$ & $48(18.4)$ \\
\hline Anosmia- $N(\%)$ & $7(2.7)$ \\
\hline Conjunctivitis- $N(\%)$ & $1(0.4)$ \\
\hline Asymptomatic- $N(\%)$ & $192(73.6)$ \\
\hline \multicolumn{2}{|l|}{ Vital signs } \\
\hline $\mathrm{SBP}(\mathrm{mmHg})$ mean $(\mathrm{SD})$ & $125.1(16.6)$ \\
\hline $\mathrm{DBP}(\mathrm{mmHg})$ mean $(\mathrm{SD})$ & $77.9(11.1)$ \\
\hline Heart Rate (bpm) mean (SD) & $84.0(11.6)$ \\
\hline Temperature (Celsius) median (IQR) & $37.1(1)$ \\
\hline Respiratory Rate (rpm) mean (SD) & $17.2(1.87)$ \\
\hline \multicolumn{2}{|l|}{ COVID-19 Category } \\
\hline Category 1: Asymptomatic & $124(47.5)$ \\
\hline Category 2: Symptomatic, No Pneumonia & $102(39.1)$ \\
\hline Category 3: Symptomatic, Pneumonia & $35(13.4)$ \\
\hline
\end{tabular}

$S B P$ systolic blood pressure, $D B P$ diastolic blood pressure, $S D$ standard deviation

ground glass opacities (GGO) were found in $1.1 \%$ of the patients (Table 3).

There is no agreement between LUS and CXR findings in detecting Covid-19 pneumonia as the Cohen's Kappa coefficient was 0.08 (95\% CI 0.06-0.22, $p=0.160$ ).

\section{Discussion}

The Ministry of Health (MOH) of Malaysia advocates admission, isolation and treatment of asymptomatic patients or those with mild symptoms in a hospital or a step-down facility rather than being quarantined at home. Dedicated COVID-19 hospitals with full intensive care support and monitoring capabilities, only accepts COVID-19 positive patients who are symptomatic and possessed features of pneumonia, either requiring supplemental oxygen or not
Table 2 Lung ultrasonography (LUS) findings of the patients included

\begin{tabular}{lll}
\hline $\begin{array}{l}\text { Lung Ultrasonography (LUS) results- } N \\
(\%)\end{array}$ & $N-261(\%)$ \\
\cline { 2 - 3 } Affected zones & Normal & Multiple B lines \\
\hline 1 (right upper anterior) & $259(99.2)$ & $2(0.8)$ \\
2 (right lower anterior) & $250(95.8)$ & $11(4.2)$ \\
3 (right upper lateral) & $256(98.1)$ & $5(1.9)$ \\
4 (right lower lateral) & $254(97.3)$ & $7(2.7)$ \\
5 (right upper posterior) & $256(98.1)$ & $5(1.9)$ \\
6 (right lower posterior) & $251(96.1)$ & $10(3.9)$ \\
7 (left upper anterior) & $260(99.6)$ & $1(0.4)$ \\
8 (left lower anterior) & $259(99.2)$ & $2(0.8)$ \\
9 (left upper lateral) & $261(100)$ & 0 \\
10 (left lower lateral) & $256(98.1)$ & $5(1.9)$ \\
11 (left upper posterior) & $257(98.5)$ & $4(1.5)$ \\
12 (left lower posterior) & $260(99.6)$ & $1(0.4)$ \\
Number of sector involvement- $N(\%)$ & $N-34(\%)$ & \\
1 sector & $20(58.8)$ & \\
2 sectors & $10(29.4)$ & \\
3 sectors & $2(5.9)$ & \\
4 sectors & $2(5.9)$ \\
LUS scoring $N$ (\%) & $N-261$ \\
Normal & $227(86.9)$ \\
Mild & $34(13.1)$ \\
\hline
\end{tabular}

Numbers do not add to $100 \%$ as some patients had more than one finding

Table 3 Chest X-ray (CXR) findings of patients included

\begin{tabular}{ll}
\hline Chest x-ray results $N(\%)$ & $N-261$ \\
\hline Type of infiltrate & \\
GGO & $3(1.1)$ \\
Consolidation & $13(5.0)$ \\
Normal & $245(93.9)$ \\
Laterality & \\
Bilateral & $9(3.4)$ \\
Centrality & \\
Central & $3(1.1)$ \\
Peripheral & $13(5.0)$ \\
Focality & \\
Multifocal & $9(3.4)$ \\
Focal & $7(2.7)$ \\
Others & \\
Pleural effusion & $2(0.8)$ \\
\hline
\end{tabular}

Numbers do not add to $100 \%$ as some patients had more than one finding

(clinical stage 3 and above) and those who are either asymptomatic or with mild symptoms but with co-morbidities [19]. This effort is done in order to risk stratify the standard of 
care to these patients and also to prevent further transmission of SARS-CoV-2 to the community.

To our knowledge this is the first study on chest imaging using LUS in out-of-hospital setting for staging COVID-19 patients. LUS was conducted as an extension to physical examination to categorize COVID-19 patients. This study focuses on the LUS findings and characteristics and their relationship with CXR images. We report that LUS detected lung pulmonary disease more frequently compared to CXR in confirmed stage 3 COVID-19 patients. Moreover, among patients with negative CXR findings, abnormalities were detected by LUS in more than half of the subjects. Multiple B-lines were the predominant findings at the right lower anterior, posterior and lateral zones which is consistent with previous case series publications [12, 20-22]. COVID-19 pneumonia predilection of affecting the right hemi-thorax has been observed in numerous literatures. Extensive literatures concluded that majority of COVID-19 pneumonia patients who had a CT thorax done were reported to have an affected right lower lobe.

Only $45.7 \%$ of stage 3 COVID-19 patients' CXR was reported as abnormal (Fig. 1). Consolidations (5.0\%) and ground glass opacities (1.1\%) were the most common findings (Fig. 4). The distribution of lesions were peripheral (5.0\%) and multifocal (3.4\%), and these findings are comparable to other studies [15, 18]. In the CXR of COVID19 patients, interstitial changes (23.7\%) and ground glass opacities $(18.9 \%)$ were the predominant descriptive findings, and the location of abnormalities were in the lower lobes (33.8\%), bilateral (20.9\%) and multifocal distributions (24.2\%). Negative chest radiographic findings alone are not adequate to rule out pneumonitis [20]. This conclusion

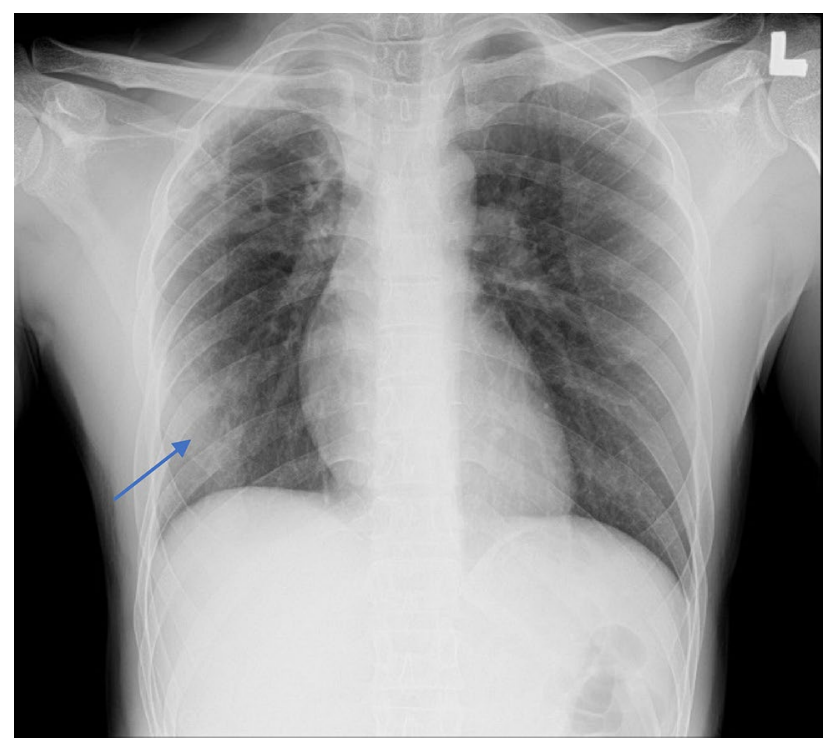

Fig. 4 Chest $\mathrm{x}$-ray (CXR) showing a ground glass opacity (GGO) over the right lower lung zone with peripheral distribution (arrow) indicates the harmful risk of over relying on CXR when making critical decisions in the management of COVID-19 patients. Another study concluded that the CXR sensitivity is about $68.1 \%$ in COVID-19 patients and the most common $\mathrm{x}$-ray findings are patchy or diffuse reticular-nodular opacities with consolidations, with basal, peripheral and bilateral location predominance [19]. A study has also shown that during the early phase of the illness, COVID-19 patients have normal chest CT and CXR, $13.8 \%$ and $41.9 \%$ respectively [23]. The majority of patients from our study had normal CXR (93.9\%) and all CXR were done early in the course of their illness. This also consistent with the earlier study. A normal CXR does not guarantee the absence of Covid-19 pneumonia, as this may be due to the difficulty of detecting early ground glass opacities on the CXR of patients presenting early in the course of their illness [20].

In our study, the lowest value and the highest value of the RALE score are 1 and 10 respectively. This shows that none of our patients deteriorated and required admission to the ICU as the RALE score has significant statistical correlation with the patient's outcome; whereby a score of more than 15 indicates the likelihood of being admitted to the ICU [19]. With LUS score of less than 8 (score 1-7) and RALE score of less than 15, all patients enrolled in this study who had evidence of COVID-19 pneumonia based on LUS were categorized under mild severity and none of the patients deteriorated at the step-down-facility nor required admission in the ICU. This may also be attributed to the incorporation of LUS in pre-hospital settings which leads to early detection of COVID-19 pneumonia and appropriate triaging and hence, proper treatment allocation that determines patients' outcome. This data also suggests better accuracy of using LUS as staging tools in out of hospital settings in determining the stages of Covid-19.

Pneumonia was once thought to be diagnosed only by using physical examinations, history taking and related methods. CXR provides certainty and is recommended as the main imaging approach for diagnosing pneumonia. Chest CT is considered the gold standard imaging approach as it provides critical information of the differential diagnoses of pneumonia [24]. However, chest CT has its limitations with high radiation exposure and cost. When CXR is replaced with LUS to diagnose pneumonia, the large decrease in the utilization of radiographs leads to a substantial overall cost reduction. Therefore, besides being portable and safe for repeat use due to zero ionizing radiation emission, LUS is also cost effective [13].

Current literature primarily focuses on CT findings, which are more sensitive than CXR and LUS but the issue of infection control in the CT suite discourages its usage. Some countries have dedicated CT suites for suspected COVID-19 patients only, which is not practical in any developing nation. Thus, portable CXR can be considered for identification 
and monitoring lung abnormalities in COVID-19 patients besides minimize the risk of cross-infection [25, 26].

Patients with chronic interstitial lung disease such as pulmonary fibrosis have a LUS pattern of multiple-B lines which is usually bilateral with irregular or fragmented pleural line which is similar to LUS in COVID-19 pneumonia. However, peripheral lung lesions are more common in COVID-19 pneumonia which is a rare finding in chronic interstitial lung disease. This poses challenges to the physicians in diagnosing patients of suspected of COVID-19 pneumonia in chronic interstitial lung disease, thus integrating with patient's medical history, physical examination and a positive RT-PCR increased the sensitivity and specificity in diagnosing patients with pre-existing lung disease $[9,10]$.

This study has several limitations. Firstly, LUS findings were not compared with chest CT scans due to limited hospital resources. Secondly, no repeated CXR was done during the course of patients' illness since majority of these patients had normal initial chest radiographs and also the difficulty to perceive ground glass opacity during early course of illness. Comparing early LUS findings with repeated CXR during the course of the illness may produce a more accurate statistical result instead. A larger cohort study of COVID-19 patients with wider spectrum of illness (from mild to moderate and severe) and the use of chest CT to compare LUS findings with would help to further define the impact of LUS when used in pre-hospital setting.

\section{Conclusion}

In conclusion, the diagnostic imaging of COVID-19 staging using LUS in out-of-hospital settings shows better detection of lung pleural disease more often than CXR for stage 3 COVID-19 patients. The study also supports the utilization of LUS to identify COVID-19 pneumonia in out-of-hospital setting.

Acknowledgements We would like to thank all the Ministry of Health staffs of Emergency and Trauma Department of both Kuala Lumpur Hospital and Sungai Buloh Hospital who have shared their wisdom, knowledge and support throughout this study.

\section{Declarations}

Funding No funding has been received.

Ethical approval and consent of approval This study was approved by Medical research and Ethics Committee (MREC) and registered with the National research register (NMRR-20-1599-55711).

Consent for publication We would like to thank the Director General of Health Malaysia for his permission to publish this article.

Competing interests The authors declare that there is no conflict of interest.
Availability of data and materials All of the data used during this study are available upon request to the corresponding author.

\section{References}

1. Zhu N, Zhang D, Wang W, Li X, Yang B, Song J, Zhao X, Huang B, Shi W, Lu R, Niu P, Zhan F, Ma X, Wang D, Xu W, Wu G, Gao GF, Tan W (2020) A novel coronavirus from patients with pneumonia in China, 2019. N Engl J Med 382:727-733

2. Yuki K, Fujiogi M, Koutsogiannaki S (2020) COVID-19 pathophysiology: a review. Clin Immunol 215:108427

3. Wu Z, McGoogan JM (2020) Characteristics of and Important Lessons from the Coronavirus Disease 2019 (COVID-19) Outbreak in China: Summary of a Report of 72314 Cases from the Chinese Center for Disease Control and Prevention. JAMA J Am Med Assoc 323:1239-1242

4. Buonsenso D, Pata D, Chiaretti A (2020) COVID-19 outbreak: less stethoscope, more ultrasound. Lancet Respir Med 8:e27

5. Aujayeb A (2020) Could lung ultrasound be used instead of auscultation? African. J Emerg Med 10:105-106

6. Pan F, Ye T, Sun P, Gui S, Liang B, Li L, Zheng D, Wang J, Hesketh RL, Yang L, Zheng C (2020) Time course of lung changes on chest CT during recovery from Coronavirus Disease 2019 (COVID-19). Radiology 295(3):715-721. https://doi.org/10. 1148/radiol.2020200370

7. Yoon SH, Lee KH, Kim JY, Lee YK (2020) Pneumonia in Korea 21:494-500

8. Zhou S, Zhu T, Wang Y, Xia LM (2020) Imaging features and evolution on CT in 100 COVID-19 pneumonia patients in Wuhan, China. Eur Radiol 30:5446-5454

9. Ma IWY, Hussain A, Wagner M, Walker B, Chee A, Arishenkoff S, Buchanan B, Liu RB, Mints G, Wong T, Noble V, Tonelli AC, Dumoulin E, Miller DJ, Hergott CA, Liteplo AS (2020) Canadian Internal Medicine Ultrasound (CIMUS) Expert consensus statement on the use of lung ultrasound for the assessment of medical inpatients with known or suspected coronavirus disease 2019. J Ultrasound Med, pp 1-14

10. Gargani L, Soliman-Aboumarie H, Volpicelli G, Corradi F, Pastore MC, Cameli M (2020) Why, when, and how to use lung ultrasound during the COVID-19 pandemic: Enthusiasm and caution. Eur Heart J Cardiovasc Imaging 21:941-948

11. Sofia S, Boccatonda A, Montanari M, Spampinato M, D'ardes D, Cocco G, Accogli E, Cipollone F, Schiavone C (2020) Thoracic ultrasound and SARS-COVID-19: a pictorial essay. J Ultrasound 23:217-221

12. Soldati G, Smargiassi A, Inchingolo R, Buonsenso D, Perrone T, Briganti D F, Perlini S, Torri E, Mariani A, Mossolani EE, Tursi $\mathrm{F}$, Mento F, Demi L. Is there a role for lung ultrasound during the COVID-19 pandemic? J Ultrasound Med. 2020.

13. Jones BP, Tay ET, Elikashvili I, Sanders JE, Paul AZ, Nelson BP, Spina LA, Tsung JW (2016) Feasibility and safety of substituting lung ultrasonography for chest radiography when diagnosing pneumonia in children: a randomized controlled trial. Chest 150:131-138

14. Soummer A, Perbet S, Brisson H, Arbelot C, Constantin JM, Lu Q, Rouby JJ (2012) Ultrasound assessment of lung aeration loss during a successful weaning trial predicts postextubation distress. Crit Care Med 40:2064-2072

15. Benchoufi M, Bokobza J, Anthony Chauvin A, Dion E, Baranne M-L, Levan F, Gautier M, Cantin D, d Humieres T, Gil-Jardine C, Benenati S, Oberlin M, Martinez M, Kahn N, Vicaut E, Bourrier P, Diallo A (2020) Lung injury in patients with or suspected COVID-19 : a comparison between lung ultrasound and chest 
CT-scanner severity assessments, an observational study medRxi 2020.04.24.20069633

16. Lichtenstein DA, Lascols N, Mezière G, Gepner A (2004) Ultrasound diagnosis of alveolar consolidation in the critically ill. Intensive Care Med 30:276-281

17. Warren MA, Zhao Z, Koyama T, Bastarache JA, Shaver CM, Semler MW, Rice TW, Matthay MA, Calfee CS, Ware LB (2018) Severity scoring of lung oedema on the chest radiograph is associated with clinical outcomes in ARDS. Thorax 73:840-846

18. Jabaudon M, Audard J, Pereira B, Jaber S, Lefrant J-Y, Blondonnet R, Godet T, Futier E, Lambert C, Bazin J-E, Bastarache JA, Constantin J-M, Ware LB (2020) Early changes over time in the radiographic assessment of lung edema score are associated with survival in ARDS. Chest 158(6):2394-2403. https://doi.org/10. 1016/j.chest.2020.06.070

19. Cozzi D, Albanesi M, Cavigli E, Moroni C, Bindi A, Luvarà S, Lucarini S, Busoni S, Mazzoni LN, Miele V (2020) Chest X-ray in new Coronavirus Disease 2019 (COVID-19) infection: findings and correlation with clinical outcome Radiol. Medica 125:730-737

20. Weinstock MB, Echenique A, Russell JW et al (2020) Chest X-ray findings in 636 ambulatory patients with COVID-19 presenting to an urgent care center: a normal chest $\mathrm{x}$-ray is no guarantee. $\mathrm{J}$ Urgent Care Med. 14(7):13-18

21. Yi Huang, Sihan Wang, Yue Liu, YaoHui Zhang, Chuyun Zheng, Yu Zheng, Chaoyang Zhang, Weili Min, MIng Yu MH (2020) A preliminary study on the ultrasonic manifestations of peripulmonary lesions of non-critical novel coronavirus pneumonia (COVID-19). https://doi.org/10.21203/rs.2.24369/v1
22. Poggiali E, Dacrema A, Bastoni D, Tinelli V, Demichele E, Ramos PM, Marcianò T, Silva M, Vercelli A, Magnacavallo A (2020) Can lung US Help critical care clinicians in the early diagnosis of novel coronavirus (COVID-19) pneumonia? Radiology 295(3):E6. https://doi.org/10.1148/radiol.2020200847

23. Guan W, Ni Z, Hu Y, Liang W, Ou C, He J, Liu L, Shan H, Lei C, Hui DSC, Du B, Li L, Zeng G, Yuen KY, Chen R, Tang C, Wang T, Chen P, Xiang J, Li S, Wang JL, Liang Z, Peng Y, Wei L, Liu Y, Hu YH, Peng P, Wang JM, Liu J, Chen Z, Li G, Zheng Z, Qiu S, Luo J, Ye C, Zhu S, Zhong N (2020) Clinical characteristics of coronavirus disease 2019 in China. N Engl J Med 382:1708-1720

24. Long L, Zhao HT, Zhang ZY, Wang GY, Zhao HL (2017) Lung ultrasound for the diagnosis of pneumonia in adults: a meta-analysis. Medicine 96(3):e5713. https://doi.org/10.1097/MD.00000 00000005713

25. Gargani L, Picano E (2015) The risk of cumulative radiation exposure in chest imaging and the advantage of bedside ultrasound. Crit Ultrasound J 7:20-23

26. Jacobi A, Chung M, Bernheim A, Eber C (2020) Portable chest $\mathrm{X}$-ray in coronavirus disease-19 (COVID-19): A PICTORIAL review. Clim Imaging 64:35-42

Publisher's Note Springer Nature remains neutral with regard to jurisdictional claims in published maps and institutional affiliations. 